\title{
Characterization of Chromobacterium violaceum pigment through a hyperspectral imaging system
}

\author{
Maria J Gallardo ${ }^{1 *}$, Juan P Staforelli ${ }^{1}$, Pablo Meza ${ }^{1,3}$, Ignacio Bordeu ${ }^{1,2}$ and Sergio Torres ${ }^{1,4}$
}

\begin{abstract}
In this paper, a comprehensive spatio-spectral and temporal analysis for Chromobacterium violaceum colonies is reported. A hyperspectral imaging (HSI) system is used to recover the spectral signatures of pigment production in a non-homogeneous media with high spectral resolution and high sensitivity in vivo, without destructing the sample. This non-contact sensing technique opens avenues to study the temporal growing of a specific section in the bacterial colony. Further, from a $580[\mathrm{~nm}]$ and 764 [nm] spatio-spectral time series, a wild-type and mutant Chromobacterium violaceum strains are characterized. Such study provides quantitative information about kinetic parameters of pigment production and bacterial growing.
\end{abstract}

Keywords: Chromobacterium; Hyperspectral systems; Real time sampling; Pigment production

\section{Introduction}

For decades, natural pigments have been extensively used in various fields of everyday life such as food production, textile industries, paper production, agricultural practices and researches, water science and technology (Arad and Yaron 1992; Sirimanne et al. 2006). Natural pigments not only have the capacity to increase the marketability of products, they also display advantageous biological activities as antioxidants and anticancer agents (Stahl and Sies 2005; Kong et al. 2010). Several intensely colored compounds have been isolated from certain microorganisms and from various environmental sources (Ahmad et al. 2012). An example is the violacein pigment, a purplecolored dye produced by one of the strains of Chromobacterium violaceum, which is an indole derivative (Duran and Menck 2001) with antitumoral, antibacterial, antiulcerogenic, antileishmanial, and antiviral activities. (Leon et al. 2001; Duran et al. 2007).

The detection of bacterial components like antibiotics, enzymes and secondary metabolites like pigments,

\footnotetext{
*Correspondence: mgallardo@cefop.udec.cl

${ }^{1}$ Center for Optics and Photonics, Universidad de Concepción, Concepción, Chile

Full list of author information is available at the end of the article
}

is important in biotechnological industries (Steele and Stowers 1991). Traditional methods are effective but can take hours or days to realize them. Besides, they are destructive as well as time consuming. On the other hand, molecular detection based techniques for bacteria identification are rapid, specific and sensitive. However, most are technically complicated and costly, and require welltrained specialists (Steele and Stowers 1991; Lazcka et al. 2007; Velusamy et al. 2010). Now, molecular spectroscopy provides less invasive or even non-destructive methods that have been proven well-suited to investigate the chemical composition of biological systems (Cen and He 2007).

Hyperspectral imaging (HSI) is an emerging technique that assimilates spectroscopy and imaging to provide both spectral and spatial information of a biological sample (Plaza et al. 2009). Further, the potential that HSIs have for in vivo optical diagnostics have being exploited due to the non-invasive feature and the massive spatio-spectral information collected by the system (Vo-Dinh 2004; Pisani et al. 2013). In this sense, HSI systems record, for each spatial location being imaged, a set of hundreds of high spectral resolution images that jointly conform the spectrum of the biological sample. Such collections of images is known as hypercube (Borengasser et al. 2008), from which the pertinent qualitative and quantitative information is

\section{是 Springer}

(c) 2014 Gallardo et al: licensee Springer. This is an Open Access article distributed under the terms of the Creative Commons Attribution License (http://creativecommons.org/licenses/by/2.0), which permits unrestricted use, distribution, and reproduction in any medium, provided the original work is properly cited. 


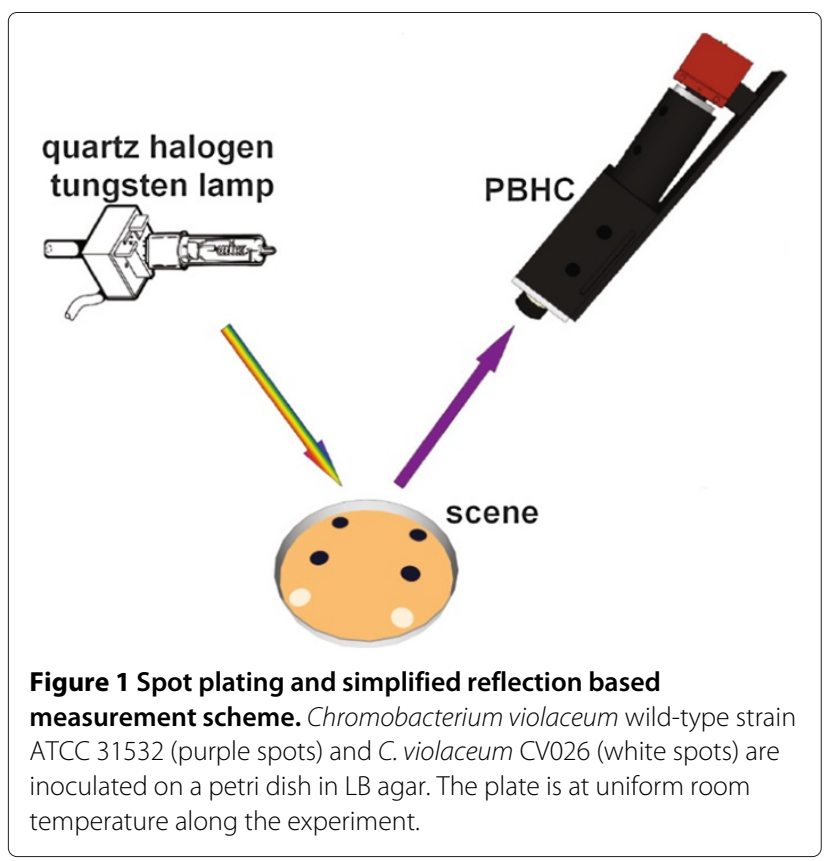

obtained (Sankaran et al. 2010; Siripatrawan et al. 2011). Different optical techniques can be applied to generate the hypercube from biological samples, some of them are based on tunable optical filters (Gat 2000), imaging spectrometers (Jun et al. 2009), and coded hyperspectral imaging (Studer et al. 2012), among other. In particular, push-broom hyperspectral cameras (PBHCs) are imaging devices that carried out the spectral decomposition by means of an optical process (Kim et al. 2010). Further, PBHCs employ a scanning procedure to record spectral information of one spatial line at a time, with the ability to scan multiple batches of samples simultaneously by moving across the process line. Also, the spatial information is important for monitoring the sample as it can be used to extract its chemical mapping at different spatial points (Zavattini et al. 2003; Singh et al. 2010). In this regard, we can use HSI system to measure the spectral signature of chemical components, like bacterial pigments, inside the cells without altering the sample and its measurements even in a heterogeneous medium where spectrophotometric signal is not resolutive.

In this work, a spectral characterization of Chromobacterium violaceum pigment through a HSI is performed from the visible spectrum to part of the near infrared spectrum $(400 \mathrm{~nm}$ to $1000[\mathrm{~nm}]$ with $1,04[\mathrm{~nm}]$ of spectral resolution). It is well known that a pigment changes the color of the reflected light as a result of selective color absorption. In this sense, the pigment is quantified associating its signal to the maximal optical absorption centered at 580 [nm] (Gerhardt, 1970; Koch, 1994). With HSI it is now possible to resolve signals and quantify the bacterial pigment with a remote, non-invasive and real-time procedure comparatively to a spectrophotometric measurement. Finally, a series of spectral signatures masking relevant information that can serve as an indicator of a specific bio-processes is obtained, i.e. pigment production and bacterial growing rate. In this case, despite using a small number of spectral bands, these are selected as a function of the amount of spectro-temporal information associated with the pigmentation process. For a different process, the evaluation of the spectral information can determine a larger number of spectral bands required for characterization. Hence, the amount of useful spectral information is directly related to the process being measured.

\section{Materials and methods \\ Bacterial cultures}

The C. violaceum wild-type strain ATCC 31532 and C. violaceum CV026 (mini-Tn5 mutant of ATCC 31532) (McClean et al. 1997) are cultured in Luria Bertani (LB) broth $(10 \%$ peptone, $5 \%$ yeast extract, $5 \% \mathrm{NaCl}$, per $1 L$

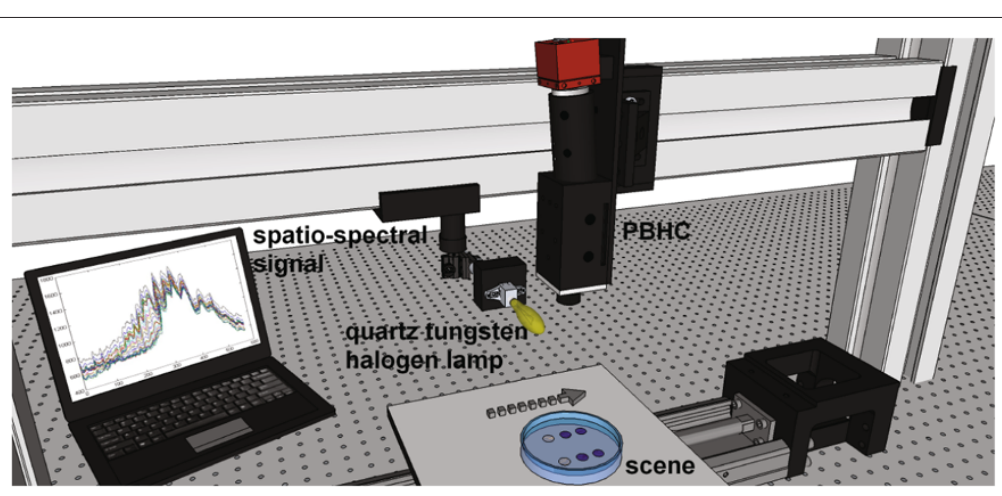

Figure $\mathbf{2}$ Laboratory prototype to retrieve the spatio-spectral image. The aluminum arc holds the illumination and camera system. The target scene lies on a movable base where the arrow indicates scan direction. Imaging is acquired during the scan process and data analysis is post-processed. 
b

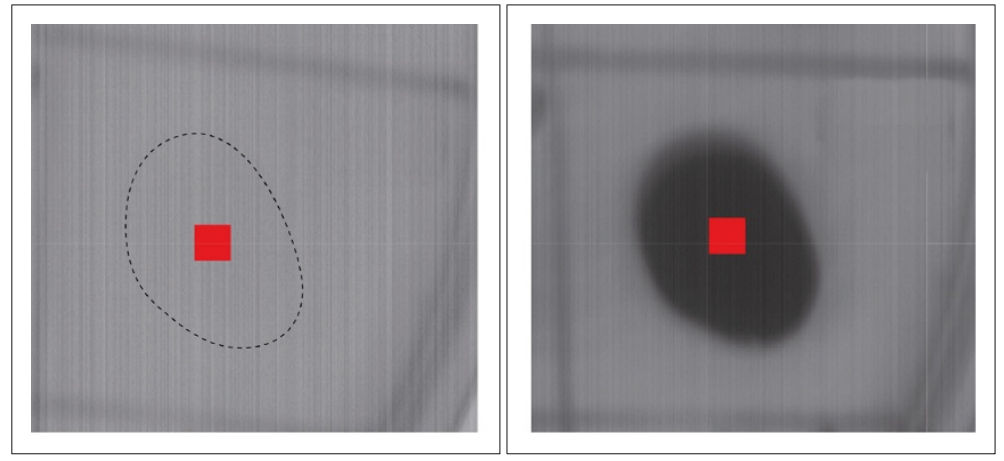

Figure 3 Selected region of interest in the reasembled spectral image at $580[\mathrm{~nm}]$ at a) $t=0 \mathrm{hrs}$ and b) $t=37 \mathrm{hrs}$.

distilled water) (Sambrook et al. 1989). Solid bacterial medium is made by the addition of agar at a final concentration of $15 \mathrm{~g} / \mathrm{L}$.

\section{Spectrophotometric measurement}

The bacterial population and the pigment production is estimated by measuring absorbance of the culture using a spectro-photometer (UV-1700 UV-VIS Spectrophotometer) at different wavelengths (350-700 [nm]). Measurements are taken each one hour during lag phase and every 30 minutes in the exponential phase. For each measurement an aliquot of liquid culture is taken and the absorbance is determined every $50[\mathrm{~nm}]$ (between 350 and $700[\mathrm{~nm}])$.

\section{Spot plating and incubation}

The initial liquid bacterial culture is grown overnight at $30^{\circ} \mathrm{C}$ in a thermo-regulated bath. Agar LB plates are inoculated with $20 \mu \mathrm{L}$ spots at pre-determined and wellspaced locations on the agar surface. We separated the wild-type strain from the mutant strain to avoid contamination. Figure 1 shows a scheme of the imaged spot

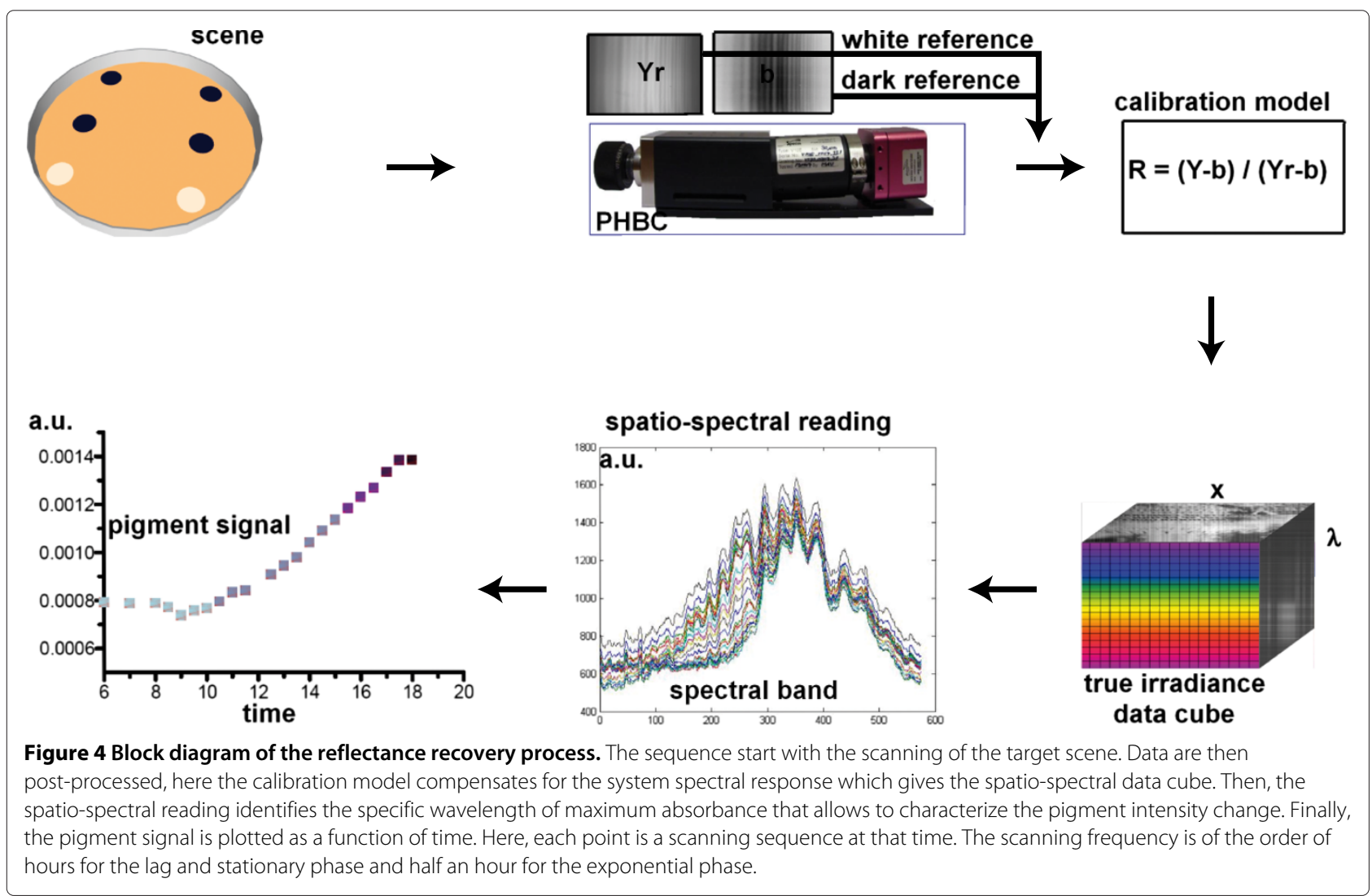


locations. The plate is incubated at room temperature in the camera stage for 24 hours. Three measurements are made, each one with four replicates of the wild-type strain and two replicates of the mutant strain.

\section{Experimental Setup}

In order to acquire image sequences with high spectral resolution, The set-up illustrated in Figure 2 is constructed, where the key component is the PBHC. Such a camera can be described, in brief, as an optoelectronic imaging system composed of four sub-systems: the optics, the spectrograph, the sensor, and the readout electronics (Borengasser et al. 2008). Unlike broadband images, in $\mathrm{PBHC}$ the spatial information is mapped onto one axis of the focal plane array (FPA), while the spectral information is mapped onto the second axis. Therefore, a biological sample (now target scene) must be scanned one line at a time by displacing the camera in an orthogonal trajectory with respect to the axis used to encode the spectral information.

The HSI system employed in this work is the Photonfocus Hurricane $40 \mathrm{~V} 10 \mathrm{E}$ PBHC. This camera is based on a CMOS FPA of $1024 \times 1024$ photo-detectors. Further, the input radiance is decomposed and converted into several spatio-spectral images through an optical process that allows us to obtain spectral bands between 400-1000 [nm], with a spectral resolution of $1.04[\mathrm{~nm}]$. Also, due to the nature of the acquisition process of $\mathrm{PBHC}$, a mobile platform has been constructed to simulate and synchronize the scanning procedure.

Furthermore, to compensate for any noise source degrading the hyperspectral data, it is required to perform calibration measurements. To this end, we have employed a quartz tungsten halogen $(\mathrm{QTH})$ calibration lamp, which guarantees an uniform and continuous spectral illumination between 200-2500 [nm]. Also, a Spectralon SRT99-120 is used as a diffuse reflectance target that ensures a reflectance of $99 \%$ between 250-2000 [nm].

As shown in Figure 2, the diffuse target lies on a petri dish holder to increase the reflectivity of the semitransparent agar and colonies. Further, during the scanning process, each line of the target scene acquired receives the same angle of illumination, then we can ensure that all spatial points were illuminated under the same conditions. The sample measurement scheme is displayed in Figure 1, where the input radiance is generated by the light reflected from the agar. Finally, the dimensions of the hypercubes produced after scanning the whole target scene are 1024 spatial pixels, 574 spectral bands, and 1000 temporal lines.

\section{Spectral reflectance recovery}

In order to retrieve the spectral reflectance of biological samples, several stages have to be performed. First, the sequence starts with the scene scanning due to the nature of the of the PBHC acquisition process. Second, the input radiance of a spatial line is decomposed and sampled into $P$ spatial pixels and $Q$ spectral bands, taking $S$ line samples at different times. Hence, the entire target scene is mapped into a hypercube of dimensions $P \times Q \times S$. Third, the hyperspectral data must be calibrated to compensate for any degrading effect produced during the signal transduction. To do so, we have mathematically represented the hyperspectral response by the following first-order model:

$$
Y\left(i, \lambda_{j}, k\right)=r\left(\lambda_{j}\right) a(i, j) R\left(i, \lambda_{j}, k\right)+b(i, j)+V(i, j, k),
$$

where the suffixes $i, \lambda_{j}$, and $k$ denote, respectively, the spatial location at the scene, the spectral band, and the temporal sample, where $i=1, \ldots, P, j=1, \ldots, Q^{\prime}$, and $k=1, \ldots, S$. The variable $R\left(i, \lambda_{j}, k\right)$ represents the input reflectance collected at the $\lambda_{j}$ th spectral band by the $i j$ th photo-detector at the $k$ th sample time. The terms $a(i, j)$ and $b(i, j)$ represent, respectively, all the multiplicative and additive noise sources corrupting the output of a PBHC. The additive term $V(i, j, k)$ is known as the temporal
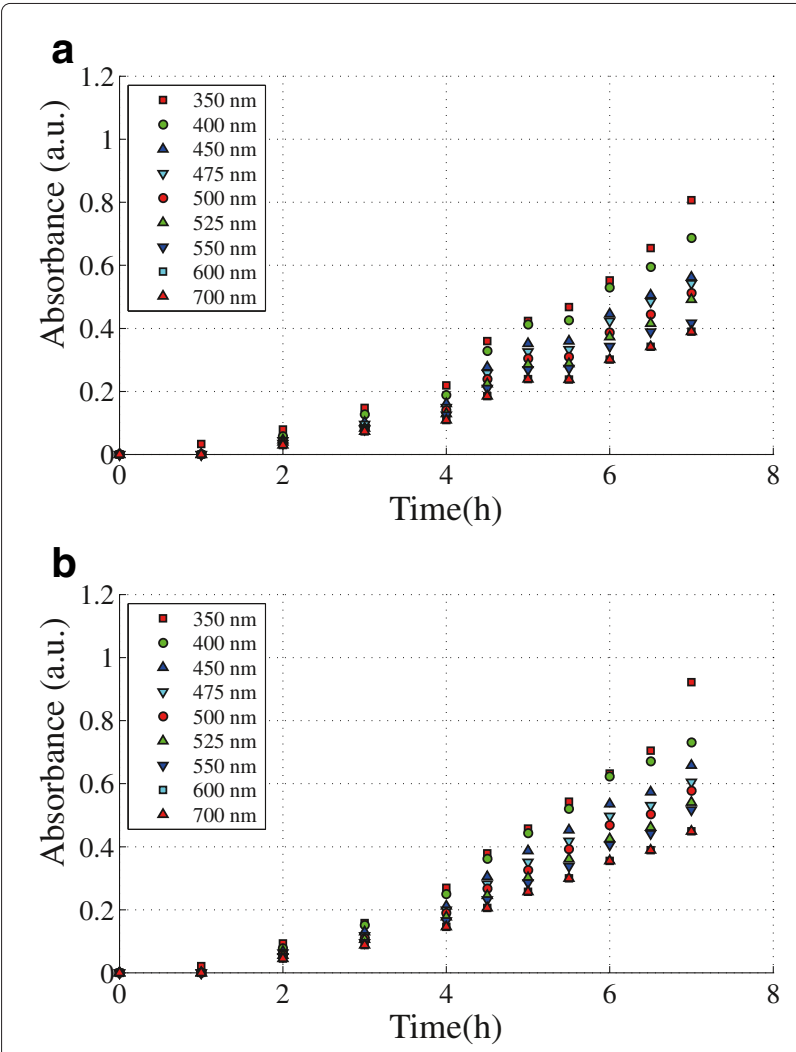

Figure 5 Spectrophotometric measurement of both C. violaceum wild-type strain (a) and C. violaceum CV026 strain (b) in liquid culture. Data shows the absorbance intensity signal versus time in the visible spectrum. 


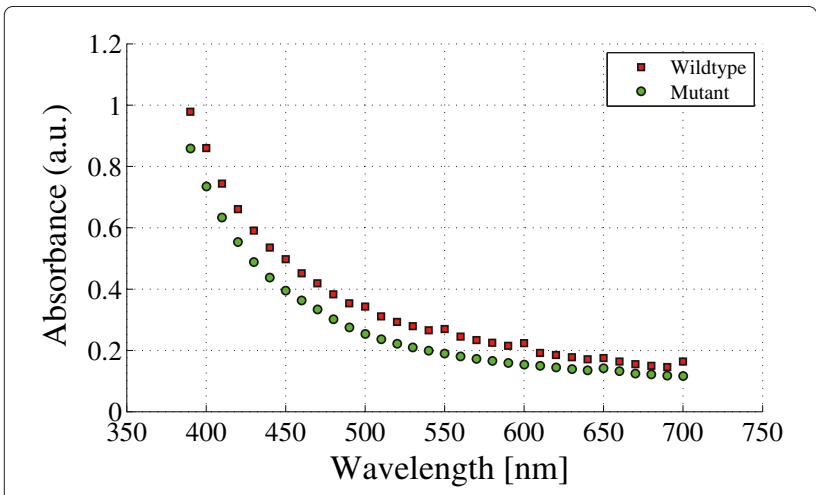

Figure 6 Spectrophotometric measurement of both C. violaceum wild-type strain and C. violaceum CV026 strain in liquid culture. Data shows the absorbance intensity signal versus wavelength. Data is taken during the stationary phase of growing, seven hours after inoculation.

white noise associated with the readout electronic for the $i j$ th photo-detector. However, in most of the cameras the white noise $V(i, j, k)$ is negligible compared to the term $b(i, j)$, so it can be disregarded. Finally, the parameter $r\left(\lambda_{j}\right)$ represents the system spectral response, i.e. the slit, the diffraction grid response and the spectral responsivity of the detector material.

In general, the additive noise term $b(i, j)$ is treated as a baseline value produced by different noise sources, i.e., dark current, non-uniformity and amplifiers noise. Such baseline can be estimated through the measurement of a dark reference. This is, considering that the spectral decomposition is carried out by an optical process, blocking the camera input prohibits the decomposition process, hence, $R\left(i, \lambda_{j}, k\right)=0$. Further, both multiplicative terms, $r\left(\lambda_{j}\right)$ and $a(i, j)$ can be estimated by means of a white reference, which is obtained by illuminating a diffuse target with the QTH calibration lamp. Consequently, the following formula is used to compensate and retrieve the spectral reflectance:

$$
R\left(i, \lambda_{j}, k\right)=\frac{Y\left(i, \lambda_{j}, k\right)-\hat{b}(i, j)}{Y_{r}\left(i, \lambda_{j}, k\right)-\hat{b}(i, j)},
$$

where $Y_{r}\left(i, \lambda_{j}, k\right)$ is the white reference value and $\hat{b}(i, j)$ is the estimated baseline obtained when measuring the dark reference. Recalling Eq. 1, by subtracting $\hat{b}(i, j)$ in Eq. 2 , the baseline value is compensated. Further, the normalization between the measured sample and the white reference compensates for the system spectral response and the term $a(i, j)$. With the spectral reflectance at hand, the absorbance values $A\left(i, \lambda_{j}, k\right)$ are retrieved and the maximal and minimal pigment absorption bands are selected, that in our case are $580[\mathrm{~nm}]$ and $764[\mathrm{~nm}]$, respectively. Therefore, to analyze the spectral behavior of the samples taken at different times, first it is required to reassemble the spatial information from the hypercube. Then, a region of interest region of interest (RI) is located on each sample, covering a spatial region equal to 21 by 21 pixels, as shown in Figure 3.

Each absorbance value enclosed by the RI is averaged to minimize any small variation produced by sensor noises or by simple irregularities. Thus, the sample averaged absorbance is calculated as follows:

$$
\bar{A}\left(i_{0}, \lambda_{j}, k_{0}\right)=\sum_{n=i_{0}-10}^{i_{0}+10} \sum_{m=k_{0}-10}^{k_{0}+10} A\left(n, \lambda_{j}, m\right)
$$

where $\left(i_{0}, k_{0}\right)$ is the RI center. It should be noted that since each pixel represents an absorbance value, selecting random measurement points is also a valid alternative.
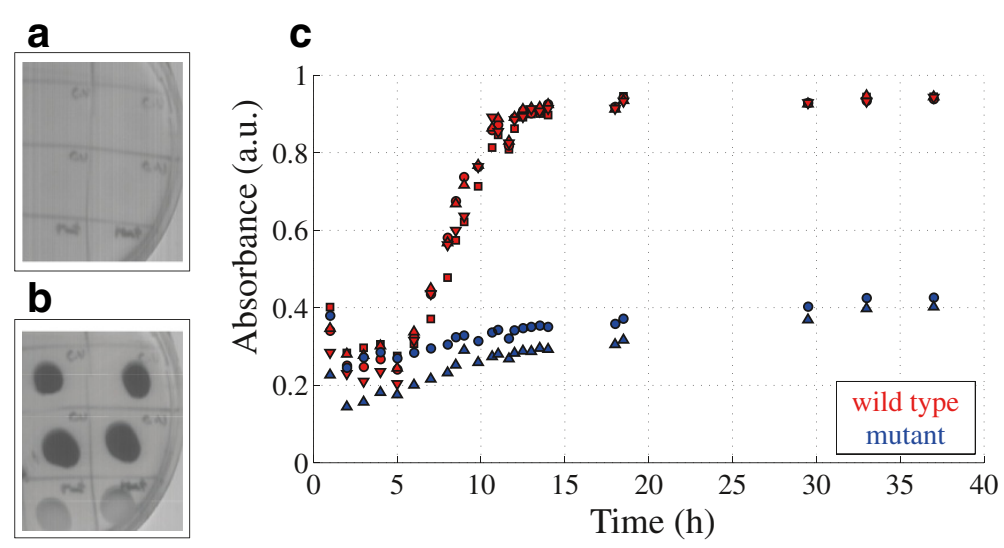

Figure 7 Imaging at $580 \mathrm{~nm}$ of both C. violaceum wild-type strain (a) and C. violaceum CV026 strain (b). Hyperspectral measurements in (c) show the absorbance intensity signal versus time for both strains. 

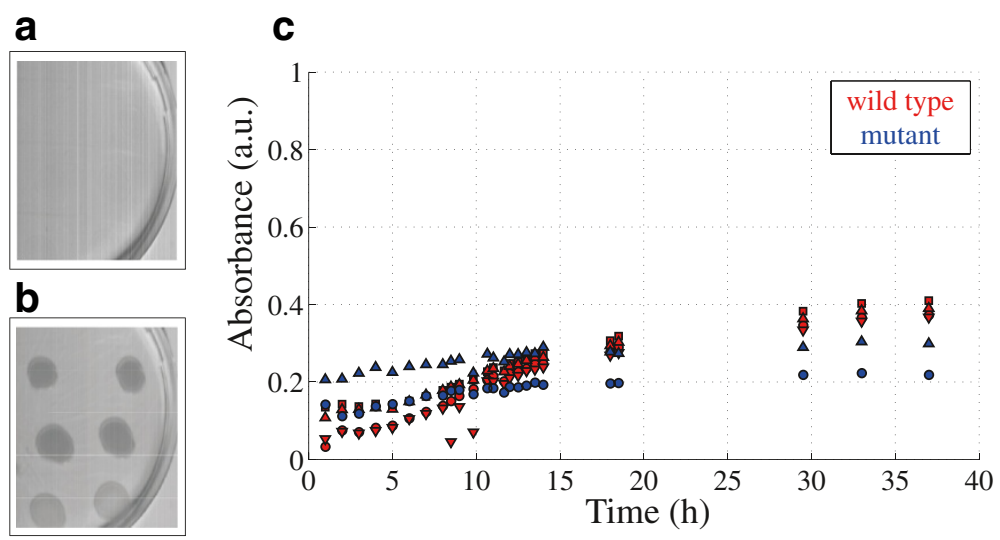

Figure 8 Imaging at 764 [nm] of both C. violaceum wild-type strain (a) and C. violaceum CV026 strain (b). Hyperspectral measurements in (c) show the absorbance intensity signal versus time for both strains.

Finally, to summarize the methodology, a block diagram explaining each stage of the reflectance recovery process is presented in Figure 4.

\section{Results}

One of the advantages of using HSI systems is to recover the spectral signatures of pigment production in turbid media with high spectral resolution and sensitivity, in contrast to optical absorption based instruments. The traditional spectrophotometer procedure disposes the sample inside small quartz containers. Here, colored laser beams creates the observed absorption spectrum. When the sample is a complex mixture of bacterial cell components and pigment, the observed spectrum is indistinguishable for a clear identification, e.g., between the $C$. violaceum wild-type strain and $C$. violaceum CV026 strain as shown in Figure 5.

Although both samples are differentiable trough simple eye-inspection, the absorbance intensity signal shown in Figure 6 is poor for quantification. This constraint is due to the screening by the turbid media itself which is a result of optical absorption in the visible spectrum by the bacterial cell components (membranes, organelles, DNA, proteins, etc.) (Gerhardt 1994; Koch 1970).

With the aim to resolve the spectral differences between the strains associated with the pigment production, we use a PBHCs that allows to obtain spatio-spectral information without disturbing the sample. The experiment is developed by plating both strains in LB-agar at equal initial cell concentration, and following the methodology explained in the previous section. In particular, two spectral bands are selected and the subsequent temporal samples have information regarding the biological process. To obtain the signal, a band centered at 580 [nm] which corresponds to the wavelength of maximal absorption, is selected. In Figure $7 \mathrm{c}$ is shown the absorbance intensity signal versus time for both strains. Now the pigment absorbance signal is successfully retrieved and is clearly differentiable between the strains. The absorbance pigment intensity signal of the $C$. violaceum wild-type strain exhibits a bacterial growing-like curve behavior.

In order to obtain quantitative information regarding the pigment production curves of Figure 7, some considerations need to be taken into account. The absorbance at $580[\mathrm{~nm}]$ has simultaneous information of pigment and bacterial cell components. Thus, a band centered at 764 [nm] which corresponds to the minimal pigment absorption, is now selected. The absorbance intensity signal versus time for both strains at $764[\mathrm{~nm}]$ can be seen in Figure 8.

The information regarding pigment and cell components can be summarized in Figure 9. Data is fitted by

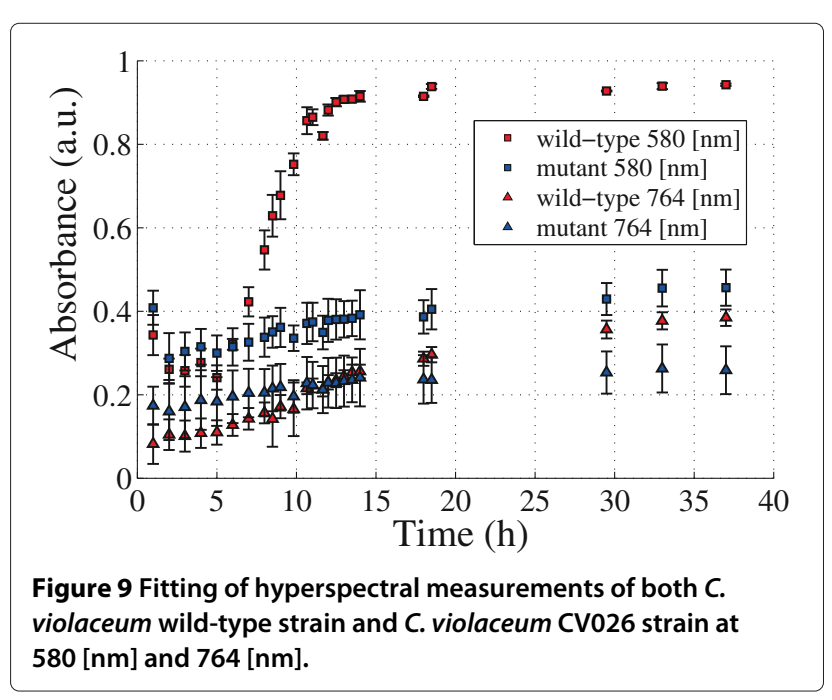


Table 1 Kinetic parameter of pigment production and bacterial growth

\begin{tabular}{|c|c|c|c|c|}
\hline \multirow[t]{2}{*}{ Hyperspectral imaging measurement ${ }^{a}$} & \multicolumn{2}{|c|}{$\begin{array}{c}\text { C. violaceum } \\
\text { wild-type strain }\end{array}$} & \multicolumn{2}{|c|}{$\begin{array}{l}\text { C. violaceum } \\
\text { CV026 strain }\end{array}$} \\
\hline & $g(h)^{c}$ & $\mathbf{k}\left(h^{-1}\right)^{c}$ & $g(h)^{c}$ & $\mathrm{k}\left(h^{-1}\right)^{\mathrm{c}}$ \\
\hline $580[\mathrm{~nm}]$ (pigment production + bacterial growth) & $5.28 \pm 1.10$ & $0.137 \pm 0.029$ & $29.77 \pm 6.85$ & $0.025 \pm 0.006$ \\
\hline 764 [nm] (bacterial growth) & $7.57 \pm 1.14$ & $0.094 \pm 0.014$ & $26.93 \pm 7.31$ & $0.028 \pm 0.008$ \\
\hline $580[\mathrm{~nm}] / 764[\mathrm{~nm}]$ (pigment production) & 15.9 & 0.043 & $d$ & $d$ \\
\hline OD measurement ${ }^{\mathrm{b}}$ & $2.93 \pm 0.65$ & $0.249 \pm 0.055$ & $8.4 \pm 1.23$ & $0.08 \pm 0.011$ \\
\hline
\end{tabular}

${ }^{\mathrm{a}}$ measurements are made on solid medium with no agitation at RT.

${ }^{\mathrm{b}}$ measurements are made on liquid medium with agitation at RT.

'The coefficients are estimated considering a confidence level of $95 \%$ that the real value is in that range.

${ }^{d}$ mutant strain does not show spectral response.

using a non-linear least squares method on the exponential phase. Kinetic parameter of this fitting are shown in Table 1.

The grown parameters $g$ and $k$ for both strains at 764 $[\mathrm{nm}]$ are calculated. At this wavelength only information of bacterial growing is obtained. Although the wild-type strain grows faster than the mutant strain both have comparable velocities $k$. This is in agreement with the values found in literature and those obtained with the spectrophotometric measurements (see Table 1).

To quantify the violacein production, a division of functions is obtained. The foregoing is achieved between the curve regarding information of the bacterial pigment and bacterial growth (Figure 9)-squares and the curve containing information only of the bacterial growth (Figure 9)-triangles (Figure 10). Comparing the velocities of mutant strains at $580[\mathrm{~nm}]$ and $764[\mathrm{~nm}]$, noted that both values are equal, as expected, due to the lack of pigment production. C. violaceum CV026 strain are does not show spectral response in the spectral range analyzed.

\section{Discussion}

The violacein production can be quantified using a spectrophotometer, after an extraction and purification process (Rettori and Duran 1998; Lu et al. 2009). This procedure fails to identify pigment remotely and not allows to follow the process in time. To follow the pigment production without destroying the bacterial cells we use a Hyperspectral Imaging System. This system provide spectral and spatial information of the Chromobacterium violaceum enabling us to obtain kinetic parameters of bacterial growth and pigment production.

The violacein production can be quantified at 580 [nm] by subtracting the contribution of the bacterial cell components at $764[\mathrm{~nm}]$ which resulted in $k_{\text {pigment }}=0.043$. Therefore, the results are in good agreement with the values calculated using optical density (OD) at 600 [nm] (see Table 1) and with those found in the literature (Corpe 1964).

In this work, a real time, non invasive and simple process to quantify bacterial pigment production trough HSI system is reported. The PBHC camera produces a hypercube

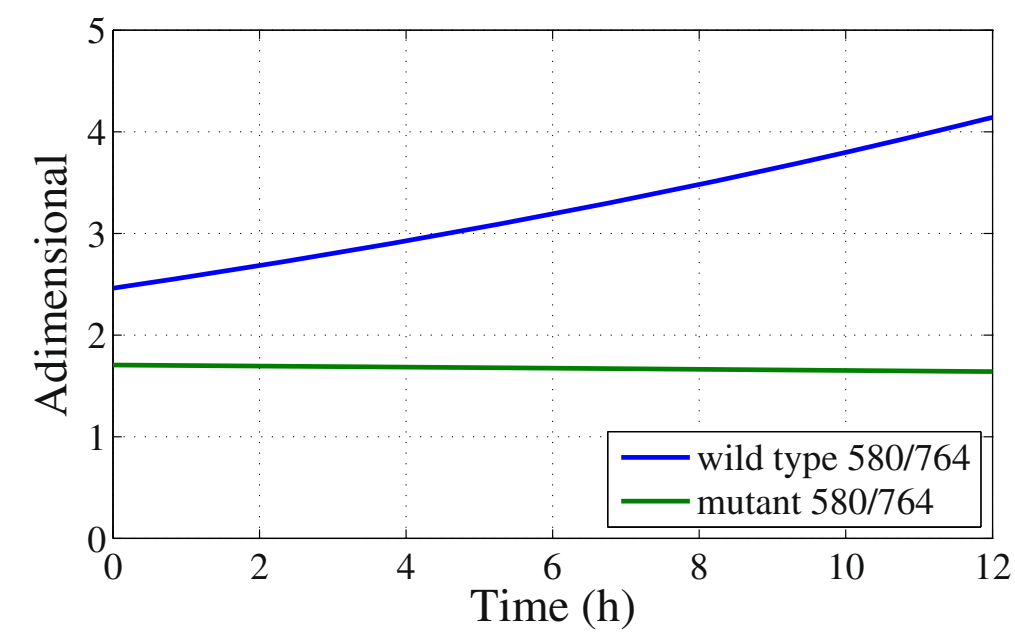

Figure 10 Mathematical division of the fitting curves generated at $580[\mathrm{~nm}]$ and $764[\mathrm{~nm}]$ of both $C$. violaceum wild-type strain and $C$. violaceum CV026 strain. 
with spatial and spectral information in function of time without destroying the sample. Hence we can analyze multiple samples with high wavelength resolution in a single experiment. Based on the process, the technique can be extensible to multiple colored samples at the same time. This optical technique could be useful for the analysis of complex samples such as bacterial biofilms, environmental samples or non-culture bacteria (Cortizo and de Mele 2000). This can be applied to quantify another biological pigments obtained from natural source without alter the environmental conditions. For example, studies can be done to quantify photosynthetic pigments, like chlorophylls or carotenoids, presents in some plants or bacteria. Also this technique can be used in the pigment determination in fruits or other exportation products. The advantage of this technique is the decomposition of the components of the biological system in a set of wavelengths each one associated to a specific spatio-temporal character. This allows to get new insights of a particular process in a complex media. Also, since we have detected the specific wavelengths that allows to characterize the bacterial pigment production and growing process, the next step is developing a filter-based imaging system with the aim to increases the temporal resolution of the measurements. That is, designing a monochromatic camera that includes a filter tuned at the wavelength of interest, discarding the scanning stage and acquiring the spatio-spectral image that exhibits greater variation in time (Nishino et al. 2013). Further, such devices do not require a scanning stage, which could increase the temporal resolution of our measurements.

\section{Competing interests}

The authors declare that they have no competing interests.

\section{Acknowledgements}

This work was supported from PIA-CONICYT PFB0824. M.J. Gallardo acknowledge support from FONDECYT 3140167. J.P. Staforelli acknowledge support from FONDECYT 11110145. Pablo Meza is supported by a CONICYT $\mathrm{PhD}$ Scholarship. Ignacio Bordeu is supported by a CONICYT MSc Scholarship.

\section{Author details}

${ }^{1}$ Center for Optics and Photonics, Universidad de Concepción, Concepción Chile. ${ }^{2}$ Departamento de Física, Universidad de Concepción, Concepción, Chile. ${ }^{3}$ Departamento de Ingeniería Eléctrica, Universidad de La Frontera, Temuco, Chile. ${ }^{4}$ Departamento de Ingeniería Eléctrica, Universidad de Concepción, Concepción, Chile.

Received: 5 November 2013 Accepted: 30 December 2013 Published: 13 January 2014

\section{References}

Ahmad WA, Yusof N, Nordin N, Zakaria ZA, Rezali MF (2012) Production and characterization of violacein by locally isolated Chromobacterium violaceum grown in agricultural wastes. Appl Biochem Biotechno 167:1220-1234. doi:10.1007/s12010-012-9553-7

Arad SM, Yaron A (1992) Natural pigments from red microalgae for use in foods and cosmetics. Trends in Food Sci Technol 3(0):92-97. doi:10.1016/0924-2244(92)90145-M
Borengasser M, Hungate WS, Watkins RL (2008) Hyperspectral remote sensing: principles and applications. CRC Press, Series Remote Sensing Applications Series: 130. doi:10.1201/9781420012606.ch3

Cen H, He Y (2007) Theory and application of near infrared reflectance spectroscopy in determination of food quality. Trends in Food Sci Technol 18(2):72-83. doi:10.1016/j.tifs.2006.09.003

Corpe WA (1964) Factors influencing growth and polysaccharide formation by strains of Chromobacterium Violaceum. J Bacteriol 88(5):1433-1441

Cortizo M, de Mele M (2000) Preliminary characterization of thin biofilms by optical microscopy. BIOFOULING 15(4):253-260. doi $=10.1080 / 08927010009386316$

Duran N, Menck C (2001) Chromobacterium violaceum: A review of pharmacological and industiral perspectives. Critic Rev Microbiol 27(3):201-222. doi:10.1080/20014091096747

Duran N, Justo GZ, Ferreira CV, Melo PS, Cordi L, Martins D (2007) Violacein: properties and biological activities. Biotechnol Appl Biochem 48(3):127-133. DOI:10.1042/BA20070115

Gat N (2000) Imaging spectroscopy using tunable filters: a review. 4056:50-64. DOl:10.1117/12.381686

Gerhardt P (1994) Methods for general and molecular bacteriology. American Society for Microbiology, Washington, DC, p 518

Jun W, Kim M, Lee K, Millner P, Chao K (2009) Assessment of bacterial biofilm on stainless steel by hyperspectral fluorescence imaging. Sensing Instrum Food Qual Saf 3(1):41-48. DOI:10.1007/s11694-009-9069-1

Kim TH, Kong HJ, Kim TH, Shin JS (2010) Design and fabrication of a 900-1700 nm hyper-spectral imaging spectrometer. Optics Commun 283(3): 355-361. DOI:10.1016/j.optcom.2009.10.022

Koch AL (1970) Turbidity measurements of bacterial cultures in some available commercial instruments. Anal Biochemis 38(1):252-259. DOI:10.1016/0003-2697(70)90174-0

Kong KW, Khoo HE, Prasad KN, Ismail A, Tan CP, Rajab NF (2010) Revealing the power of the natural red pigment lycopene. Molecules 15(2):959-987. DOI:10.3390/molecules15020959

Lazcka O, Campo FJD, Munoz FX (2007) Pathogen detection: A perspective of traditional methods and biosensors. Biosensors and Bioelectronics 22(7): 1205-1217. DOI:10.1016/j.bios.2006.06.036

Leon LL, Miranda CC, Souza AOD, Durán N (2001) Antileishmanial activity of the violacein extracted from Chromobacterium violaceum. J Antimicro Chemotherapy 48(3):449-450. DOI:10.1093/jac/48.3.449

Lu Y, Wang L, Xue Y, Zhang C, Xing XH, Lou K, Zhang Z, Li Y, Zhang G, Bi J, Su Z (2009) Production of violet pigment by a newly isolated psychrotrophic bacterium from a glacier in Xinjiang, China. Biochem Eng J 43(2):135-141

McClean KH, Winson MK, Fish L, Taylor A, Chhabra SR, Camara M, Daykin M, Lamb JH, Swift S, Bycroft BW, Stewart GSAB, Williamsl P (1997) Quorum sensing and Chromobacterium violaceum: exploitation of violacein production and inhibition for the detection of $n$-acylhomoserine lactones, Microbiol 143(12):3703-3711. DOI:10.1099/00221287-143-12-3703

Nishino K, Nakamura K, Tsuta M, Yoshimura M, Sugiyama J, Nakauchi S (2013) Optimization of excitation-emission band-pass filter for visualization of viable bacteria distribution on the surface of pork meat. Opt Express 21(10):12,579-12,591. DOI:10.1364/OE.21.012579. http://www.opticsexpress.org/abstract.cfm?URI=oe-21-10-12579

Pisani M, Zucco M, Caricato V, Egidi A (2013) Hyperspectral imaging: a tool for biological measurements. In: Filtz J-R, Larquier B, Claudel P, et Favreau J.-O (eds) 16th International Congress of Metrology, EDP Sciences, p 14007. doi $=10.1051 /$ metrology/201314007, http://dx.doi.org/10.1051/ metrology/201314007

Plaza A, Benediktsson JA, Boardman JW, Brazile J, Bruzzone L, Camps-Valls G, Chanussot J, Fauvel M, Gamba P, Gualtieri A, Marconcini M, Tilton JC, Trianni G (2009) Recent advances in techniques for hyperspectral image processing. Remote Sensing Environment 113 Supplement 1(0): S110- S122. DOI:10.1016/j.rse.2007.07.028

Rettori D, Duran N (1998) Production, extraction and purification of violacein: an antibiotic pigment produced by Chromobacterium violaceum. World J Microbiol Biotechnol 14(5):685-688

Sambrook J, Fritsch E, Maniatis T (1989) Molecular Cloning: a laboratory manual. 2nd edition. Cold Spring Harbor Laboratory Pr, N.Y., Cold Spring Harbor Laboratory

Sankaran S, Ehsani R, Etxeberria E (2010) Mid-infrared spectroscopy for detection of huanglongbing (greening) in citrus leaves. Talanta 83(2):574-581. DOl:10.1016/j.talanta.2010.10.008 
Singh CB, Jayas DS, Paliwal J, White ND (2010) Identification of insect-damaged wheat kernels using short-wave near-infrared hyperspectral and digital colour imaging. Comput Electron Agric 73(2):118-125. DOI:10.1016/j.compag.2010.06.001

Sirimanne P, Senevirathna M, Premalal E, Pitigala P, Sivakumar V, Tennakone K (2006) Utilization of natural pigment extracted from pomegranate fruits as sensitizer in solid-state solar cells. J Photochemis Photobiol A: Chemis 177(2-3):324-327. DOI:10.1016/j.jphotochem.2005.07.003

Siripatrawan U, Makino Y, Kawagoe Y, Oshita S (2011) Rapid detection of Escherichia coli contamination in packaged fresh spinach using hyperspectral imaging. Talanta 85(1):276-281. DOl:10.1016/j.talanta.2011.03.061

Stahl W, Sies H (2005) Bioactivity and protective effects of natural carotenoids. Biochimica et Biophysica Acta (BBA) - Molecular Basis of Disease 1740(2): 101-107. DOl:10.1016/j.bbadis.2004.12.006

Steele DB, Stowers MD (1991) Techniques for selection of industrially important microorganisms. Ann Rev Microbiol 45(1):89-106. DOl:10.1146/annurev.mi.45.100191.000513

Studer V, Bobin J, Chahid M, Mousavi HS, Candes E, Dahan M (2012) Compressive fluorescence microscopy for biological and hyperspectral imaging. Proc Nat Acad Sci 109(26):E1679-E1687. DOl:10.1073/pnas.1119511109

Velusamy V, Arshak K, Korostynska O, Oliwa K, Adley C (2010) An overview of foodborne pathogen detection: In the perspective of biosensors. Biotechnol Adv 28(2):232-254. DOl:10.1016/j.biotechadv.2009.12.004

Vo-Dinh T (2004) A hyperspectral imaging system for in vivo optical diagnostics. Eng Med Biol Mag, IEEE 23(5):40-49. DOI:10.1109/MEMB.2004.1360407

Zavattini G, Vecchi S, Leahy R, Smith D, Cherry S (2003) A hyperspectral fluorescence imaging system for biological applications In: Nuclear Science Symposium Conference Record. 2003 IEEE, pp 942-946, Vol.2. DOI:10.1109/NSSMIC.2003.1351850

doi:10.1186/2191-0855-4-4

Cite this article as: Gallardo et al:: Characterization of Chromobacterium violaceum pigment through a hyperspectral imaging system. AMB Express 2014 4:4.

\section{Submit your manuscript to a SpringerOpen ${ }^{\circ}$ journal and benefit from:}

- Convenient online submission

- Rigorous peer review

- Immediate publication on acceptance

- Open access: articles freely available online

- High visibility within the field

- Retaining the copyright to your article

Submit your next manuscript at $\boldsymbol{\nabla}$ springeropen.com 